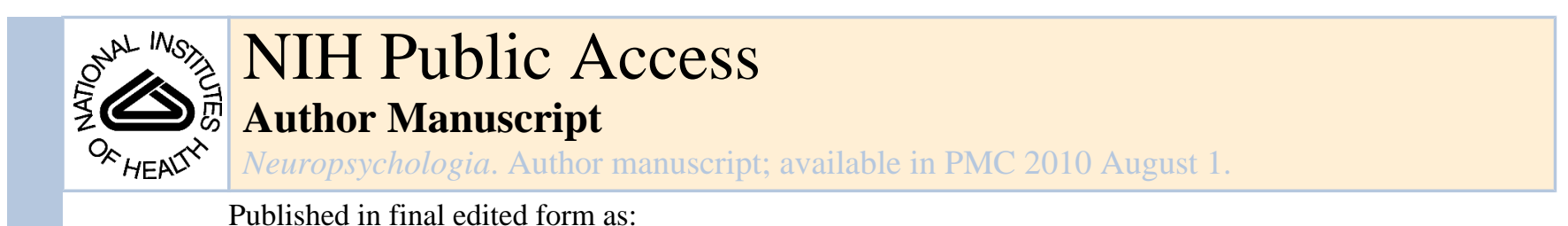

Published in final edited form as:

Neuropsychologia. 2009 August ; 47(10): 2015-2028. doi:10.1016/j.neuropsychologia.2009.03.004.

\title{
Cognitive Reserve
}

\author{
Yaakov Stern \\ Cognitive Neuroscience Division of the Taub Institute, and the Departments of Neurology and \\ Psychiatry, Columbia University College of Physicians and Surgeons
}

The concept of reserve has been proposed to account for the disjunction between the degree of brain damage or pathology and its clinical manifestations. For example, a head injury of the same magnitude can result in different levels of cognitive impairment, and that impairment can vary in its rate of recovery. Similarly, several prospective studies of aging have reported that up to $25 \%$ of elders whose neuropsychological testing is unimpaired prior to death meet full pathologic criteria for Alzheimer's disease (Ince, 2001), suggesting that this degree of pathology does not invariably result in clinical dementia. As will be described in detail below, many studies indicate that a set of life experiences such as educational and occupational exposure and leisure activities are associated with reduced risk of developing dementia and with a slower rate of memory decline in normal aging. Cognitive reserve (CR) postulates that individual differences in the cognitive processes or neural networks underlying task performance allow some people to cope better than others with brain damage. This paper attempts to produce a coherent theoretical account of reserve in general and of cognitive reserve in particular. It then reviews some of my group's epidemiologic and imaging research that has lent support to the concept of cognitive reserve and helped elucidate its neural underpinnings. It should be stressed that this review is focused on my group's work, and is not a thorough review on the entire literature on the topic.

Because my work has focused on aging and dementia, I will discuss CR's relation to these brain changes. The concept of CR, however, is applicable to almost any situation where brain function is disrupted. Thus, for example, proxies for higher CR have also been reported to mediate incidence of dementia in HIV (Farinpour et al., 2003), as well as cognitive changes associated with schizophrenia, bipolar disorder and depression (Barnett, Salmond, Jones, \& Sahakian, 2006), and traumatic brain injury (Kesler, Adams, Blasey, \& Bigler.E.D., 2003).

\section{Brain Reserve and Cognitive Reserve}

Reserve can be roughly classified into passive and active models. Brain reserve (Katzman, 1993) is an example of a passive model, where reserve derives from brain size or neuronal count. Larger brains can sustain more insult before clinical deficit emerges, because sufficient neural substrate remains to support normal function. This approach to reserve has been codified in the threshold model (Satz, 1993), which revolves around the construct of "brain reserve capacity". The model recognizes that there are individual differences in brain reserve capacity. It also presupposes that once brain reserve capacity is depleted past some fixed critical threshold specific clinical or functional deficits emerge. Thus, individual differences in brain reserve

Correspondence to Yaakov Stern, Taub Institute, $630 \mathrm{~W} 168^{\text {th }}$ Street, New York, NY 10032. Telephone: 212-342-1350, Fax:

212-342-1838, Email: ys11@ columbia.edu.

Publisher's Disclaimer: This is a PDF file of an unedited manuscript that has been accepted for publication. As a service to our customers we are providing this early version of the manuscript. The manuscript will undergo copyediting, typesetting, and review of the resulting proof before it is published in its final citable form. Please note that during the production process errors may be discovered which could affect the content, and all legal disclaimers that apply to the journal pertain. 
capacity lead to differences in the clinical expression of a particular degree of damage to the brain.

There are several reasons why threshold models can be termed passive models of reserve. First, this type of model assumes that there is some fixed cutoff or threshold below which functional impairment will occur for everyone. In the case of AD, this threshold might be depletion of synapses to the point where only a specific number remain. Second, threshold models are essentially quantitative models. They assume that a specific type of brain damage will have the same effect in each person, and that repeated instances of brain damage sum together. Individuals differ only in their overall brain capacity, and brain damage is either sufficient or insufficient to deplete brain reserve capacity to some critical level. Threshold models do not account for individual differences in how the brain processes cognitive or functional tasks in the face of the disruption caused by brain damage.

In contrast to passive models of reserve, active models such as CR suggest that the brain actively attempts to cope with brain damage by using pre-existing cognitive processes or by enlisting compensatory processes (Stern, 2002). Although two patients might have the same amount of brain reserve capacity, the patient with more CR may tolerate a larger lesion than the other patient before clinical impairment is apparent. Thus, an active model does not assume that there is some fixed cutoff or threshold at which functional impairment will occur. Rather it focuses on the processes that allows individuals to sustain brain damage and maintain function.

As will be discussed below, I have suggested that the possible neural implementation of cognitive reserve be subdivided into two components, neural reserve and neural compensation. Neural reserve refers to inter-individual differences in cognitive processing that exist in the normal healthy brain. Neural compensation refers to alterations in cognitive processing that may take place in order to cope with brain pathology. Table 1 summarizes the working definitions for brain reserve, cognitive reserve and its subcomponents.

It has become clearer in recent years that the demarcation between brain reserve and cognitive reserve is not clear cut. First, from a strict point of view, the differences in cognitive processing envisioned by the cognitive reserve model must also have a physiologic basis, in that the brain must ultimately mediate all cognitive function. The difference is in terms of the level of analysis. Presumably, the physiologic variability subsumed by cognitive reserve is at the level of variability in synaptic organization, or in relative utilization of specific brain regions. Thus cognitive reserve implies anatomic variability at the level of brain networks, while brain reserve implies differences in the quantity of available neural substrate. Second, many of the factors associated with increased cognitive reserve, such as cognitively stimulating experiences, have a direct effect on the brain. The child developmental literature suggests that not only do individuals with higher IQ have larger brain volume (Willerman, Schultz, Rutledge, \& Bigler.E.D., 1991) (Kesler et al., 2003), but that cognitively stimulating aspects of life experience may also be associated with increased brain volume. It is also now clear that stimulating environments and exercise promote neurogenesis in the dentate gyrus (Brown et al., 2003; van Praag, Shubert, Zhao, \& Gage, 2005). Both exercise and cognitive stimulation regulate factors that increase neuronal plasticity (such as BDNF) and resistance to cell death. Finally, there is evidence to suggest that environmental enrichment might act directly to prevent or slow the accumulation of AD pathology (Lazarov et al., 2005). Thus, a more complete account of $\mathrm{CR}$ would have to integrate these complex interactions between genetics, the environmental influences on brain reserve and pathology, and the ability to actively compensate for the effects of pathology. 


\section{Measures of Reserve}

For brain reserve, anatomic measures such as brain volume, head circumference, synaptic count, or dendritic branching are effective measures of reserve. Based on the epidemiologic evidence reviewed below, variables descriptive of lifetime experience are commonly used as proxies for CR. These include measures of socioeconomic status, such as income or occupational attainment, educational attainment and leisure activity. In some populations, degree of literacy might be a better marker for CR than number of years of formal education because it is a more direct measure of educational attainment (Manly, Touradji, Tang, \& Stern, 2003; Manly, Schupf, Tang, \& Stern, 2005). Finally, specific measured attributes have been used as indices of reserve, particularly IQ.

Education might also be a marker for innate intelligence, which may in turn be genetically based or a function of exposures. Some studies suggest that an estimate of IQ, or premorbid IQ might actually be a more powerful measure of reserve in some cases (Albert \& Teresi, 1999; Alexander et al., 1997). Still, education and other life experiences have been shown to impart reserve over and above that obtained from innate intelligence. Studies have demonstrated separate or synergistic effects for higher educational and occupational attainment and leisure activities, suggesting that each of these life experiences contributes independently to reserve (Evans et al., 1993; Mortel, Meyer, Herod, \& Thornby, 1995; Rocca et al., 1990; Stern et al., 1994; Stern et al., 1995). A prospective study showed that estimated IQ at age 53 was separately influenced by childhood cognition, educational attainment and adult occupation (Richards \& Sacker, 2003). These observations stress that CR is not fixed; at any point in one's lifetime it results from a combination of exposures.

\section{Epidemiologic Evidence for CR}

Figure 1 illustrates the way in which cognitive reserve may mediate between AD pathology and its clinical expression. We assume that AD pathology slowly increases over time, and this is graphed on the $\mathrm{x}$-axis. The $\mathrm{y}$-axis represents cognitive function, in this case memory performance. $\mathrm{AD}$ pathology probably begins to develop many years before the disease is expressed clinically and slowly becomes more severe. At some point this developing pathology will begin to produce the initial cognitive changes associated with dementia. This is labeled as the point of inflection in the figure. The pathology will subsequently result in symptoms of sufficient severity to allow the clinical diagnosis of AD. The reserve model predicts that because there are individual differences in reserve capacity, there will be individual differences in the amount of pathology required for the initial expression of clinical symptoms and the subsequent diagnosis of disease. Note that in the figure individuals with more reserve have more AD pathology when dementia is diagnosed. Therefore, all other things being equal the disease should emerge later in people with higher CR. This leads to the prediction that the rate of incident dementia should be lower in individuals with higher cognitive reserve.

In 1994, we reported incident dementia data from a follow up study of 593 community-based, non-demented individuals aged 60 years or older (Stern et al, 1994)). After 1 to 4 years of follow up, 106 became demented; all but five of these met research criteria for AD. The risk of dementia was increased in subjects with low education, where the relative risk (RR) of developing dementia over the follow-up period was 2.2 times higher in individuals with less than 8 years of education than in those with more education (95\% confidence interval [CI], 1.33 to 3.06). Similarly, risk of incident dementia was increased in those with low lifetime occupational attainment (RR, $2.25 ; 95 \% \mathrm{Cl}, 1.32$ to 3.84 ). Risk was greatest for subjects with both low education and low lifetime occupational attainment (RR, 2.87; 95\% CI, 1.32 to 3.84).

To the extent that aspects of educational and occupational attainment reflect lifetime exposures that would increase CR, it would be logical to expect that environmental exposures later in life 
would also be beneficial. Thus, we assessed participation in a variety of leisure activities characterized as intellectual (e.g., reading, playing games, going to classes) or social (e.g., visiting with friends or relatives etc) in a population sample of non-demented elderly in New York (Scarmeas, Levy, Tang, Manly, \& Stern, 2001). During follow-up, subjects who engaged in more of these activities had $38 \%$ less risk of developing dementia. Interestingly, specific classifications of leisure activity (such as purely intellectual activities) did not provide better prediction then a simple summation of all the considered activities.

A review paper (Valenzuela \& Sachdev, 2005) found 22 papers reporting cohort studies of the effects of education, occupation, premorbid IQ and mental activities in incident dementia published up to 2004. Ten out of 15 studies demonstrated a significant protective effect of education; 9 out of 12 a protective effect of occupational attainment; 2 out of 2 a protective effect of premorbid IQ; and 6 out of 6 a protective effect of engaging in leisure activities. Studies that did not find a protective effect had the lowest dementia rates. Integrating these studies, the authors reported that higher reserve was associated with significantly lowered risk for incident (i.e. newly developed) dementia. The summary odds ratio, 0.54 (95\% CI, 0.49 to 0.59 ), indicates a decrease in risk of $46 \%$ in individuals with high reserve.

Note that this incidence approach involves implicit assumptions that the presence and rate of decline of disease pathology is independent of CR. Further, in this and most approaches to studying CR, disease pathology is not measured. It is simply inferred that reduced incidence of dementia is evidence of the effect of CR on the clinical expression of the pathology. Finally, because the diagnosis of $\mathrm{AD}$ relies heavily on cognitive testing, and higher education or IQ is associated with better performance on most tests, one must be careful to avoid confounding the predictors and outcomes in analyses such as these.

There is also evidence for cognitive reserve in studies of age-related cognitive decline. In an ethnically diverse cohort of nondemented elders in New York City, we found that increased literacy (presumably associated with quality and extent of education) was associated with slower decline in memory, executive function, and language skills (Manly et al., 2003). Several other studies of normal aging reported slower cognitive and functional decline in individuals with higher educational attainment (Albert et al., 1995; Butler, Ashford, \& Snowdon, 1996; Chodosh, Reuben, Albert, \& Seeman, 2002; Christensen et al., 1997; Farmer, Kittner, Rae, Bartko, \& Regier, 1995; Snowdon, Ostwald, \& Kane, 1989; Lyketsos, Chen, \& Anthony, 1999; Colsher \& Wallace, 1991). These studies suggest that the same education-related factors that delay the onset of dementia also allow individuals to cope more effectively with brain changes encountered in normal aging.

In contrast to the studies reviewed above, in which greater reserve was associated with better outcomes, a series of studies of patients with AD have suggested that, once AD emerges, those with higher reserve have poorer outcomes. In a prospective study of AD patients matched for clinical severity at baseline (Stern, Tang, Denaro, \& Mayeux, 1995), patients with greater education or occupational attainment died sooner than those with less attainment. We also found that higher education was associated with more rapid cognitive decline in patients with prevalent AD (Stern, Albert, Tang, \& Tsai, 1999) and subsequently replicated this observation in patients with incident AD (Scarmeas, Albert, Manly, \& Stern, 2006). Similarly, we have noted more rapid decline in cognitive function in AD patients who engaged in more leisure activities prior to dementia onset (Helzner, Scarmeas, Cosentino, Portet, \& Stern, 2007). Notably, in the latter two studies cognitive decline was more rapid in patients with higher CR both immediately before and after the incidence of $\mathrm{AD}$.

Although these findings might appear counterintuitive at first, their theoretical basis is illustrated in Figure 1. Because people with higher CR can tolerate more AD pathology, 
memory function will begin to be affected later in time, after more pathology has accumulated. Thus, the "point of inflection" where memory begins to decline will occur later in patients with higher CR. Another assumption is that at some point AD pathology must become too severe to support the processes that mediate either CR or memory function. The timing of this final common endpoint will be the same in all patients regardless of their level of CR. Given these two assumptions, it follows that the time between the point of inflection and complete loss of function will be shorter in patients with higher CR. This leads to the prediction that memory decline after the inflection point must be more rapid in patients with higher CR. The figure also indicates a point in time when dementia is diagnosed. Note that dementia is diagnosed later (i.e. when pathology is more advanced) in individuals with higher CR although memory performance is of comparable severity. Thus in AD patients of comparable clinical severity, memory decline should be more rapid in those with higher CR. Because advanced AD pathology is associated with conditions that lead to death, this theoretical model can also explain our observation of more rapid time to death in AD patients with higher CR.

While this theoretical model explained our epidemiologic findings, there was no direct evidence for the model until a recent study by Hall et al (Hall et al., 2007). They examined memory test data collected at regular intervals from healthy elders who were followed prospectively until they became demented. They modeled the data to determine the point inflection at which memory began to decline more rapidly. They found that the point of inflection occurred later in patients with higher education, and that the rate of memory decline after the point of inflection was more rapid in those with higher education.

\section{Evidence for CR From Studies of Regional Cerebral Blood Flow}

Our first imaging studies of $\mathrm{CR}$ were designed to test the hypothesis that at any given level of clinical AD severity an individual with a higher level of CR should have greater AD pathology. The logic behind this prediction is demonstrated in figure one. Note that, after the point of inflection, at any particular memory test score on the x-axis, pathology (on the y-axis) is more advanced in patients with higher CR. In these studies, we used resting regional cerebral blood flow ( $\mathrm{rCBF}$ ) as a surrogate for $\mathrm{AD}$ pathology. This is based on observations that specific regional $\mathrm{rCBF}$ changes in $\mathrm{AD}$ are related to the underlying $\mathrm{AD}$ pathology and $\mathrm{rCBF}$ becomes lower as the pathology advances (DeCarli et al., 1992; Friedland, Brun, \& Bundinger, 1985; McGeer, McGeer, Harrop, Akiyama, \& Kamo, 1990). In AD patients matched for clinical severity (as assessed with measures of cognition and function), we found negative correlations between resting rCBF and years of education (Stern, Alexander, Prohovnik, \& Mayeux, 1992), such that higher education was associated with more depleted flow specifically in parietotemporal areas that are affected in AD. These findings imply that patients with higher education can tolerate more AD pathology than those with lower education and still appear clinically similar.

In a subsequent analysis of the same subjects we found a similar inverse relationship between rCBF and occupational attainment, even after controlling for educational attainment, suggesting that some aspects of occupational experiences imparted reserve over and above that obtained from education (Stern et al., 1995). We used data from the Dictionary of Occupational Titles to characterize occupational demands and found that two features of occupation were protective in this model. The first was interpersonal skills, which reflects the degree to which a job requires interaction with people vs. machines. The second was physical demands, which is consistent with the subsequent observations that physical exercise is beneficial for cognition. Interestingly, we originally had predicted that the substantive complexity of occupation would be the protective feature. However, this aspect of occupational demands correlated very strongly with education and, therefore, did not contribute significantly in this model. In a later O15 PET study, we replicated our initial observations and also extended the findings to leisure 
activities (Scarmeas et al., 2003a): we found an inverse relationship between $\mathrm{rCBF}$ and increased engagement in leisure activities, even after controlling for educational and occupational attainment. These observations have been replicated several times by other groups as well (Alexander et al., 1997; Perneczky et al., 2006).

The implications of these imaging findings were confirmed in a prospective clinical study with subsequent neuropathological analysis. Education was found to modify the association between AD pathology assessed post mortem and levels of cognitive function proximate to death: for the same degree of brain pathology there was better cognitive function with each year of education (Bennett et al., 2003).

\section{Neural Mechanisms Underlying CR}

The epidemiologic and $\mathrm{CBF}$ at rest data provide evidence for the existence of CR. However, they cannot provide clues as to the neural mechanisms that may mediate CR. To pursue this question, my group turned to cognitive activation studies using O15 PET and fMRI.

I have suggested that the neural implementation of CR might take two forms: neural reserve and neural compensation (see Table 1) (Stern et al., 2005). The idea behind neural reserve is that there is natural inter-individual variability in the brain networks or cognitive paradigms that underlie the performance of any task. This variability could be in the form of differing efficiency or capacity of these networks, or in greater flexibility in the networks that can be invoked to perform a task. While healthy individuals may invoke these networks when coping with increased task demands, the networks could also help an individual cope with brain pathology. An individual whose networks are more efficient, have greater capacity, or are more flexible might be more capable of coping with the disruption imposed by brain pathology.

Neural compensation refers to the process by which individuals suffering from brain pathology use brain structures or networks (and thus cognitive strategies) not normally used by individuals with intact brains in order to compensate for brain damage. I reserve the term neural compensation for a situation where it can be demonstrated that the more impaired group is using a different network than the unimpaired group. Distinguishing between these two possible neural implementations of CR is the necessary starting point for designing, analyzing and interpreting functional imaging studies in this area.

\section{Exploring the Neural Mechanisms Underlying Cognitive Reserve}

In this section I will review the reasoning underlying the different approaches my group has taken when exploring the neural underpinnings of CR. In the next section I will review studies that demonstrate these approaches. In order to provide focus, the discussion and reviewed studies will focus on exploring CR in normal aging. For the most part, the questions to be asked in neuroimaging studies when exploring the neural basis of cognitive reserve in aging mirror those addressed in most neuroimaging studies of cognition in aging. The major addition is the focus on individual variability, and on how that variability relates to proxies for cognitive reserve such as education, IQ, occupation, and leisure activities.

Figure 2 summarizes the hierarchy of questions that must be addressed in neuroimaging studies of CR. As will be discussed below, each of these questions is not trivial, and addressing these questions requires many task design and analytic considerations. The remainder of this section is devoted to explicating the research questions described in the figure. Before doing so, however, it must be stressed that this scheme assumes that the neural implementation of cognitive reserve can be explored by observing similarities and differences in task-related activation -- i.e. differences in how specific tasks are implemented. Above and beyond this scheme, it is possible that cognitive reserve may be mediated by networks that are unrelated 
to those typically revealed during activation studies. That is: cognitive reserve may be mediated by a generic network that is not task-specific and can be elicited during the performance of many tasks. This idea would be consistent with the concept that cognitive reserve helps maintain effective function in the face of brain pathology across a wide range of activities. I will return to this idea in the final set of imaging studies reviewed below.

\section{Are the networks underlying task performance the same in young and old?}

Addressing the question of whether task-related activation in two groups is the same or different is complicated by inter-related conceptual, task design and analytic issues. These reflect the fact that, for any task, a network underlying task performance can differ in its expression as a function of its efficiency or capacity. Any condition that affects brain function, including normal aging, can have an impact on network efficiency or capacity, complicating the interpretation of group or individual differences in the degree of task-related activation.

Efficiency and capacity are most readily discussed when considering task demand. For example, assume the same brain network is activated by young and old during the encoding phase of a verbal memory task. If a young and an old group are given a 5-word memory task, this task might be trivial for the young subjects, but demanding for the older subjects. That is, the network might be more efficient in the younger subjects such that less activation of the network might be associated with similar or superior performance to that seen in the older group. Thus, for this simple task, greater activation might be seen in old people than in young people. Conversely, a network might have greater capacity in the younger group; that is, it is capable of activating to a greater degree in the young than the old subjects under conditions of greater demand. Thus, in a 20-word memory task, greater activation might be seen in the young subjects than in the old. This potential relationship between task demand, efficiency and capacity is illustrated in Figure 3 (from (Stern, 2007). On the $X$ axis is increasing task demand, referring to a within-subject manipulation wherein the difficulty of the task is increased in a parametric manner. The $\mathrm{Y}$ axis represents task-related activation either at one particular brain location or throughout some brain network. The figure demonstrates hypothesized curves relating task demand to task-related activation in young and old individuals. The rate of rise in the curve is an index of the efficiency of the system. Thus, in this hypothetical example, the rate of rise is much slower in the young subjects than in the older subjects, consistent with the idea that the brain network is more efficient in the younger subjects. The asymptote of the curves could indicate the capacity of the system. In these hypothetical curves, the brain network has a greater capacity in the younger subjects than in the older subjects, suggesting that it might continue to respond effectively in the face of increased task demand. Note in the illustration that at relatively low task demand, greater activation might be seen in old people than in young people. Conversely, at greater task demand, greater activation might be seen in the young subjects than in the old.

Because of these considerations, different levels of task-related activation across groups in and of itself is very difficult to interpret. Using the same task at different levels of difficulty, one might find greater or less activation in any brain area in the young vs. older subjects. However, these differences do not necessarily indicate that the two groups are using different brain networks to mediate task performance.

Imaging investigators typically attempt to account for these considerations in their study design. Often investigators will attempt to match task performance in the young and old groups. This might be done through careful subject selection, or by manipulating the task demands themselves so that subjects perform at a comparable level. For example, in some of the studies described below, my group titrated task difficulty in each individual subject in order to ensure that each subject performed the task at a comparable accuracy level. However, this approach does not really solve the problem. First, even with the most careful matching procedures, it is 
probably not truly possible to ensure comparable performance in each subject or group; groups might be matched on one measure, such as performance accuracy, but still differ on another, such as reaction time. Second, matching on performance across groups or individuals does not really account for possible mismatches in the underlying efficiency or capacity of the networks mediating that performance. Because of these considerations, my group has begun to use tasks where task demand can be parametrically varied within each subject. This allows one to observe how task-related activation changes over a range of task difficulty, and to compare these demand/activation functions in young and old subjects.

Image analysis approaches must also be adjusted to take these efficiency capacity issues into account. Voxel-based analyses of imaging data as traditionally applied are not sufficient to discriminate between situations where the neural network underlying task performance differs in two groups and those where the network is actually the same but is operating at different efficiency and capacity. Figure 4, from a paper by Zarahn et al (Zarahn, Rakitin, Abela, Flynn, \& Stern, 2007) illustrates this concept. Note that groups A and B express identical brain networks, but to different degrees. This is a reasonable expectation if, for example, an older group expressed the same brain network as a younger group, but with less efficiency. However, when comparing thresholded maps between groups, one might incorrectly conclude that activation of voxel 3 is unique to group A. One solution to this problem is to use analytic approaches that investigate spatial covariance patterns as opposed to comparing groups on a voxel-by-voxel basis. Appropriate implementations of covariance approaches can directly assess whether or not two groups' task-related activation can be described by a single spatial pattern. With appropriate study designs, voxel-based analyses can also address these issues. One possible approach, reviewed below, is to examine how task-related activation relates to some other variable, such as performance or CR. If the relationship differs in young and old individuals, then one can reasonably conclude that there is some difference in how these areas mediate performance in the two groups.

In summary, a key step in exploring the neural basis of cognitive reserve is to determine whether the old and young groups use the same or different networks during task performance. Two techniques that could be useful in avoiding the inherent complications in comparing young and old are parametric control of task demand and the use of spatial covariance rather than voxelbased analyses. If the two groups use the same network, then the next step might be to explore whether this network differs across the groups in its efficiency or capacity.

\section{Efficiency, Capacity and CR}

The idea that network efficiency or capacity might be related to cognitive reserve stems directly from the concept of neural reserve. As described above, the concept of neural reserve is that inter-individual variability in the brain networks that underlie task performance of a healthy brain might make some people more capable than others of coping with the destruction imposed by brain pathology. One straightforward way that these individual differences could be expressed would be in the form of differential efficiency or capacity. Therefore, even in young healthy individuals without any age- or AD-related pathology, differences in network efficiency and capacity might be identified, and these differences might be associated with proxies for CR. Such an association would suggest that neural reserve is mediated in part by differential network efficiency and capacity.

A testable working hypothesis is that a person with higher $\mathrm{CR}$ will show patterns of task-related activation that are more efficient and have higher capacity. A straightforward measure of efficiency is the amount of task-related activation as a function of performance or task load. A more efficient network will show less activation in order to produce the same (or better) level of performance. This prediction is illustrated in Figure 3, where the young individuals show slower increase in expression as task demand increases. Similar differences in efficiency 
might be noted within young or old subjects as a function of CR. Capacity might be characterized by the ability to activate a network at high load levels. This is again illustrated in Figure 3, where the young individuals show greater network expression at higher levels of task demand. Again, we might hypothesize that a person with high CR will have greater capacity than one with low CR such that there will be greater network expression under conditions of high load; this higher expression would presumably be accompanied by better task performance.

Clearly, there are other ways that task processing can differ in healthy individuals beyond efficiency and capacity. For example, some individuals may have greater flexibility in approaching the task or use entirely different solution strategies than others. We have focused on efficiency and capacity because these concepts are relatively straightforward to address using functional imaging.

\section{Do elders who use the alternate network to a greater degree perform better?}

If we determine that the networks underlying task performance differ in young and old, we are in a position to address the concept of neural compensation. When two groups process a task differently, the differentiation can take many forms. The older group could use a completely different set of brain areas (or network) than the younger group. Alternately, the older group might continue to use the same brain areas, but the relationship between activation in these areas could be reorganized. Finally, the older group could continue to use the same areas as the unimpaired group, but also recruit some new areas not used by the younger group.

The definition that I have offered for neural compensation is purposely vague -- it only requires that differences between young and old subjects exist. That is because there are at least two ways that compensation may manifest itself.

The most straightforward form of compensation occurs when greater use of the alternate network by elders is associated with better performance. In this situation, we can hypothesize that this alternate network has been recruited to compensate for age-related neural changes, and that those capable of recruiting it to a greater degree are coping better with these changes. This form of compensation is consistent with the HAROLD model put forward by Cabeza (Cabeza, 2002), where better performing elders recruit additional brain areas, typically in the contralateral hemisphere from those typically recruited by younger subjects. Others have also reported examples of compensatory reallocation without the proviso that this be limited to the contralateral hemisphere. This form of compensation is also intuitively compatible with the cognitive reserve concept, in that elders who can recruit alternate solution strategies should be able to cope better with brain pathology.

While this form of compensation, which I call "compensation to improve performance" in Figure 2, has been observed in many studies, other forms of compensation are clearly possible. In the figure, these are labeled "compensation to maintain performance," In one alternate scenario, elders will recruit compensatory networks not used by young subjects, and also, as a group, perform more poorly that younger subjects. This form of compensatory activation has been noted by many investigators (Grady, Maisog, Horwitz, \& et al, 1994;Reuter-Lorenz, 2002; Madden et al., 1999). Another related scenario is that elders who recruit compensatory networks perform more poorly than elders who do not recruit these networks. Such a situation could occur in the following way: Age-related changes begin to affect the typical network used by younger individuals. At some point this network can no longer adequately support performance, so an alternate network is recruited. While this alternate network may support continued performance it may not be as optimal as the primary network, and elders who are required to use this network would perform more poorly. A simple analogy is the use of a cane, 
which allows an elder to walk but not as well as an elder who does not require a cane. In this scenario, elders who use the alternate network to a greater degree will perform more poorly.

The concept of compensation to maintain performance must be pitted against that of dedifferentiation. The concept of dedifferentiation suggests that regional processing specification decreases with aging due to increased levels of noise or decreased levels of functional integration (Li, Lindenberger, \& Sikstrom, 2001) (Rajah \& D'Esposito, 2005). When we have a situation where individuals who are performing more poorly activate areas that better performers do not, dedifferentiation is clearly a reasonable explanation. As the thoughtful discussion by Craik (Craik, 2006) emphasizes, there is no simple solution to resolving this question. However, there are some approaches and observations that may be useful. First, it might be useful to observe that dedifferentiation, as it is defined, must in some way be a result of age-related changes to the brain. As such, dedifferentiation might at times represent a compensatory response to age-related changes, in that the brain is attempting to maintain function in the face of impairments. A more empirical approach to addressing the compensation versus dedifferentiation question requires operationalizing key concepts in order to develop testable questions. For example, if an alternate network is used to compensate for the effects of age-related changes on the primary network, it may be possible to quantify these age-related changes using proxy measures such as atrophy or white matter hyperintensities. One would then predict that individuals with greater atrophy would be more likely to use the alternate network. Similarly, techniques such as transcranial magnetic stimulation (TMS) will be helpful in testing these ideas by allowing direct manipulation of brain areas or networks. Such analyses will require complex integration of multiple modalities. One concrete example of an attempt at such an analysis using gray matter atrophy as an index of age-related neural changes is presented in the review of studies below.

\section{Review of Imaging Studies}

In this section, I will review studies conducted by my group that address the questions and apply the approaches discussed above. These studies were not designed to definitively identify the neural substrates of CR. The goals are more limited. First, we wanted to develop methodology to address some of the difficulties in studying CR discussed above. Second, we wanted to explore techniques for supporting the concepts of neural reserve and neural compensation. Several data sets were analyzed more than once in order to address different theoretical issues. For convenience, the reviewed studies are listed in Table 2. This table summarizes the subjects, task, and analytic approach in each study, as well as the primary question addressed. This table also points out instances where the same data set was incorporated into different analyses. In order to simplify presentation, the review will be preceded by a general summary of study methods, with particular focus on the structure and rationale behind activation tasks and analytic approaches used in all of the studies.

\section{Imaging Study Methods}

Subjects-All papers described in this review studied healthy young and elderly adults. Young participants were typically ages 20-35, and elders typically ages 60-85 (although specific ranges vary across studies). All subjects were right handed, and carefully screened for medical or psychiatric conditions or medications that could influence task performance or the imaging measures. Elders were carefully screened to rule out dementia or mild cognitive impairment (MCI).

Activation Tasks-We used two types of activation tasks. Both were chosen to address the issues related to task difficulty discussed above. In both cases the strategy is to examine the change in activation as task becomes more difficult. This allows us to explore issues of 
efficiency and capacity. Evaluating neural responses to increased task demand has intuitive appeal, since CR is expected to be associated with the ability to cope with this demand.

Nonverbal serial recognition task: This was our first attempt to implement a task that dealt with the possibility of differential task difficulty in young and old. The task consists of the serial presentation of a set of shapes (designed to be difficult to verbally encode), followed by a series of the same number of recognition probes. For each probe, the subject uses a button press to indicate whether or not they had just seen the item. There are two task conditions. In the low demand condition, each study item is followed by a recognition probe. In the titrated demand condition, subjects study a longer list of items, and then respond to an equally long set of recognition probes. Prior to scanning, the study list size of the titrated demand condition is adjusted for each subject, such that recognition accuracy was $75 \%$. We examine the change in task-related activation from the low to the titrated demand condition. The titration procedure was intended to match task difficulty (as operationalized by recognition accuracy) across subjects. Our reasoning was that once task difficulty is equated across individuals old and young subjects, any differences in task-related activation would not be a function of differential task difficulty but would capture true alterations in how the task is processed. In other words, since we were controlling for task difficulty, any group differences would have a better chance of representing neural compensation.

Letter and Shape Sternberg Task: We selected this task because it has three conditions that systematically vary task demand or load within each subject. Thus, in contrast to the nonverbal recognition task which attempts to fix task difficulty, in this task our strategy is to examine group differences in task-related activation as task demand increases. The task is based on one published originally by Rypma, et al (Rypma, Prabhakaran, Desmond, Glover, \& Gabrieli, 1999). In the verbal version of this task, subjects study 1, 3, or 6 letters for 3 seconds (stimulus phase) followed by a 7 second retention period. They are then presented with a single probe letter and are asked to indicate whether that letter is a member of the previously studied set. In the shape version of the task, 1, 2, or 3 shapes (designed to be difficult to verbally encode) are presented at the encoding phase and a single shape is presented at probe. One straightforward behavioral measure taken from both of these tasks is the increase in reaction time associated with making a decision as set size increases. This slope of the reaction time over set size (RT slope) is considered to be a measure of working memory scanning speed. The primary aspect of fMRI data that we examined was aspects of task-related activation that changed systematically as set size (i.e. the number of letters or shapes) increased.

\section{Analytic Approaches}

General Linear Model (GLM): This is a relatively standard, voxel-based approach implemented in SPM. In the analyses described here, the primary use of the GLM approach is to examine how change in task-related activation across task load is related to measures of CR. For example, in the letter Sternberg task, load increases from one to three to six letters. The GLM approach can search for voxels where changes in task-related activation across task load correlate with proxies for CR such as IQ.

Multivariate Linear Model (MLM)(Worsley, Poline, Friston, \& Evans, 1997; Zarahn et al., 2007): This is a covariance analytic approach that can address the inherent problems with voxel-based comparisons illustrated in Figure 4. Although this approach has many applications, one primary use of this technique is to determine whether patterns of task-related activation are the same or different across groups (such as young and old subjects). Instead of looking for significant activation at each voxel using the standard statistical thresholds, this approach attempts to identify spatial patterns of covarying task-related activation across the entire brain. It can then test whether these patterns are similar or different across groups. This technique 
begins with a contrast of interest for each group, derived from the general linear model approach. The MLM analysis performs singular value decomposition on the spatially whitened contrasts of interest. Sequential latent root testing, using a global F-test is used to determine the number of significant spatial patterns. To follow the example above, in several analyses we considered load-related changes in activation during the different phases of the letter Sternberg task in both young and old subjects. The MLM analysis would begin with contrasts that separately summarize load-related change in activation at each voxel for young and old individuals. MLM uses a singular value decomposition (SVD) followed by sequential latent root testing to systematically assess how many significant latent spatial patterns are present in the imaging data. In this example, because the analyses considers 2 contrasts, one for young and one for old, it can be used to determine whether the pattern of task related activation is similar or different in the two groups. If only one significant pattern is found, this indicates that both young and old are using the same pattern. If two patterns are found, the first pattern generally represents aspects of task-related activation that are common to the two groups, and the second pattern consists of aspects of activation that differ between the two groups. Each subject's expression of spatial patterns can be quantified, and can be related to aspects of task performance.

Ordinal Trend Canonical Variates Analysis (OrT CVA) (Habeck et al., 2005a): This is another form of covariance analysis that is applied to task designs where there are systematic changes in task demand across conditions. Again, one example of such a task is the letterSternberg, where task load increases from one to three to six letters. This analysis attempts to identify a covariance pattern (or "brain network") whose expression increases as task load increases for as many individual subjects as possible. As with MLM, each subject's expression of a covariance pattern can be quantified and related to aspects of task performance. In the examples below, this technique is used to examine individual differences in efficiency within a group.

\section{Imaging Studies}

Efficiency and CR in Young Adults-As discussed above, the concept of neural reserve is not limited to older subjects. The variability in efficiency or capacity of a network should also be noted in healthy young subjects. Two studies address this issue. In one analysis (Table 2 , Study 1), we focused on the fMRI data from 40 young subjects performing the LetterSternberg task (Habeck et al., 2005b). The goal was to identify a brain network (i.e. covariance pattern) that was responsive to the increased difficulty associated with increasing the set size to be remembered from 1 to 3 to 6 letters. In individuals where this network was more efficient, the network should show less of an increase in expression as the task became more difficult. Imaging data were analyzed using Ordinal Trend Canonical Variates Analysis (OrT CVA). Using this approach, we identified a covariance pattern whose expression increased during the retention phase systematically with set size in almost all subjects. Brain regions that concomitantly increased in activation for the majority of subjects were found mainly in lateral PFC (BA 9,44), the parietal lobe (BA 7,40), anterior cingulate (BA 32), and cerebellum.

Decreasing activation was found in the occipito-temporal lobe (BA 19,39,22), insula (BA 13) as well as the medial prefrontal cortex (BA 9,10) and limbic areas (BA 24,33). Individuals with larger increases in pattern expression across the three set sizes also had larger increases in their RT as set size increased, suggesting a link between processing efficiency as measured by RT slope and network expression: individuals with greater efficiency, as indicated by lower RT slope, require a smaller increase in network expression as set size increases. Further, change in network expression across set size also correlated negatively with scores on a measure that estimates IQ, the National Adult Reading test (NART)(Nelson \& O'Connell, 1978; Grober \& Sliwinski, 1991). In other words, individuals with higher IQ demonstrated greater efficiency in network expression. This demonstrates that there are individual differences in network 
efficiency even in healthy young adults, and that CR might be associated with greater efficiency. This finding is supportive of the concept of neural reserve.

Another study of healthy young adults (Table 2, Study 2) also suggested a link between network inefficiency and lower CR. We analyzed the data from seventeen young subjects who underwent fMRI while performing our nonverbal serial recognition task using OrT CVA (Habeck et al., 2003). Recall that this task has two conditions, low demand (1 shape to be studied and recognized) and titrated demand (the number of shapes to be studied and recognized pre-adjusted such that recognition accuracy $=75 \%$ ). Again the goal was to evaluate a network which showed increased expression as the subject moved from the easier to the harder task. During the study phase of the task (i.e., when subjects were viewing the shapes to remember them later), we identified a covariance pattern that showed a systematic change in expression from the low to the titrated demand condition in 15 of the 17 subjects. A set of brain regions showed covarying decreases in expression; these included Brodmann area 7 (precuneus), anterior cingulate gyrus, bilateral thalamus, right insula, right middle temporal gyrus, and bilateral inferior frontal gyrus, perhaps consistent with a network modulating spatial attention. The key finding for the discussion here was that the larger the increase in any subjects's network expression from low to titrated demand condition, the lower his NART IQ. That is, subjects with lower CR showed the largest increases in expression of this brain network across the two difficulty conditions. Interestingly, the same network was operative during the test phase as well, and showed the same relationship to CR. We conclude from this that this network is less efficient in individuals with lower CR.

These two studies provide good examples of the concept of neural reserve. In both, higher CR was associated with greater network efficiency. One might hypothesize that individuals with these more efficient networks might cope better with age-related neural changes.

Are Patterns Of Activation The Same In Young And Old?-Several studies focused on the basic question of whether young and old subjects use the same neural networks during task performance. In one study (Table 2, Study 3), we used Multivariate Linear Modeling (MLM) to compare fMRI data of 40 young and 18 old subjects acquired during performance of the Letter-Sternberg task (Zarahn et al., 2007). The analyses presented here focus on loadrelated networks: networks that showed increased expression with increasing load (i.e. as the number of letters to be remembered increased). Recall that MLM allows us to determine whether or not spatial patterns of load-related activation are the same or different in the young and old groups. We found a load-related brain network used during the encoding phase that did not differ in old and young subjects. Similarly, a load-related network used during the recognition (or probe) phase of the task did not differ across the two groups. As outlined in Figure 2, this allows us to follow up with analyses of differential efficiency or capacity of the networks in young and old. These analyses are addressed in the section below. In contrast, we found that during the retention phase of the task there were two spatial patterns, one expressed by both young and old subjects and the second only by the older subjects. Again as described in Figure 2, this finding of differences in the activation in young and old prompts us to explore whether or not these differences represent compensatory activation in the elders. This question is explored in the section below entitled "Different networks underlying task performance in young and old."

We followed the same process in the analysis on data from young and old performing the shape Sternberg task (Table 2, Study 4) (Holtzer et al., 2008). As with the letter Sternberg task, we found that load-related activation during the stimulus and probe phases could each be described in both the young and old subjects by a single spatial pattern. Again, this indicates young and old individuals used similar brain networks during the performance of these task phases, prompting us to explore differential efficiency and capacity of thee networks across young and 
old subjects. These findings are reported below. During the retention phase of the task, two spatial patterns were noted, indicating the aspects of the neural substrate for this phase of the task differ in young and old.

Another study used a different analytic approach to determine whether the networks underlying task performance differed in young and old (Table 2, Study 8) (Scarmeas et al., 2003b). This PET study used the nonverbal serial learning task with its two conditions, low and titrated demand. The cognitive reserve variable that we used in this study was a factor score that summarized years of education and scores on two IQ indices, the NART and WAIS-R vocabulary score. Using a GLM approach, we began by searching for voxels in which there was a correlation between the CR measure and the change in activation from the low to the titrated condition. We found such areas in both the young and old subjects. The more crucial analysis from a theoretical point of view was to search for areas in which the relationship between task-related activation and cognitive reserve differed in young and old subjects (i.e a load by group interaction). For example, in a voxel in the cingulate gyrus young adults with higher CR showed increased activation from the low to the titrated demand condition; i.e., the relationship between task-related activation and CR was positive. However in the old subjects, the relationship between activation and $\mathrm{CR}$ at this same location was negative: those with higher CR showed smaller increases in activation. If we assume that people with more CR are doing a task in a more optimal manner, then the positive relationship in the young would suggest that it is more adaptive to show increased activation at this brain location as a task gets more difficult. However, the older subjects with higher CR are doing exactly the opposite. This finding suggests that there has been some reorganization of the networks underlying CR in the old subjects versus the young subjects. We hypothesize this represents a reorganization in the elders subjects driven by age-related neural changes in the older group. This change in network utilization would be a candidate for neural compensation. However, the compensation for aging here is not in how the task itself is done but rather in the aspects of the task-related activation that differ as a function of CR.

Effect of Aging on Efficiency and Capacity-As outlined in Figure 2, once we determine that old and young are using the same network(s) to mediate task performance, we can follow up by asking whether network efficiency and capacity are comparable in the two groups. As described above, in Study 3, 40 young and 18 old individuals were imaged while performing the letter Sternberg task. Using MLM to examine spatial patterns of task-related activation, we determined spatial patterns used during the stimulus and probe phases of the task did not differ in young and old. Since both groups used the common networks during these task phases, we could address the question of whether there were age-related differences in network efficiency or capacity. We calculated a measure of neural inefficiency: the ratio of the amount of performance-relevant computational work performed (operationalized here with measures of behavioral performance such as the increase in RT with increased set size) per unit network expression. Evaluation of expression of the common network noted during the stimulus phase determined that this network was more inefficient in the elder subjects. That is, as the task got harder elders increased network expression to a greater degree than young subjects but benefitted less from the use of the network in terms of performance. This is a demonstration of how age-related neural changes can limit the efficiency of a network, while the network itself remains unchanged. In contrast, a similar analysis did not find differential inefficiency during the probe phase. We saw no evidence for capacity limitation of elder vs. younger subjects in either the stimulus or probe phase. That is, the older group was capable of activating the networks to the same degree as the younger subjects.

We conducted a similar analysis of data from young and old performing the shape Sternberg task (Table 2, Study 4) (Holtzer et al., 2008). This task is more challenging than the letter Sternberg because it uses novel shapes as stimuli rather than highly familiar letters. As with 
the letter Sternberg task, we found that load-related activation during the stimulus and probe phases could each be described in both the young and old subjects by a single spatial pattern. Again, this indicates that young and old individuals used similar brain networks during the performance of these task phases. We found no evidence for differences in network efficiency during the stimulus or probe phase. However, we did find that expression of the network identified during the probe phase was greater in the young than in the old subjects. Thus, in this case, the better performance by the younger subjects was accompanied by increased expression of the underlying brain network. This suggests a capacity difference, with the younger subjects able to activate the common network to a greater degree than the older subjects. The fact that the shape Sternberg task could elicit a difference in capacity while the letter Sternberg task did not should not be surprising as the shape task is much more demanding and, indeed, is more likely to stress the capacity of even young subjects.

\section{Different networks underlying task performance in young and old}

As outlined in Figure 2, when we determine that young and old use different networks during task performance, we can follow up by exploring whether the altered activation patterns in the old are compensatory. For example, in the study that we have been discussing of 40 young and 18 old subjects on the letter Sternberg task using MLM (Table 2, Study 3) (Zarahn et al., 2007), we found that load-related activation during the retention phase of the task was characterized by two spatial patterns. The first pattern was used by both young and old subjects, and consisted of areas often associated with working memory. Regions whose expression increased with memory load included midline cerebellum, left insula/inferior frontal gyrus, left hippocampus, right middle/superior frontal gyri, left inferior/middle frontal gyri into the pre-central gyrus, left inferior/superior parietal lobule, right cingulate gyrus into medial/ superior frontal gyri and left medial/superior gyri. Regions where signal decreased with increased working memory included midline cingulate, left medial/superior temporal gyri, right medial frontal gyrus and left cingulate gyrus. In contrast, the second pattern was used only by the older subjects; mean expression of this pattern in the younger subjects did not differ significantly from zero. The only brain area in this pattern to reach statistical significance was the right parahippocampal gyrus. Interestingly, in the older subjects there was a negative correlation between activation of this additional network and overall task performance (as assessed by RT slope) - subjects who used the additional network more performed worse. No such relationship was observed in the younger subjects.

We considered two alternate explanations for this observation. One might argue that since the more the older subjects use this second network the poorer they perform, use of this network cannot be considered compensatory, and that its use is consistent with dedifferentiation. An alternate view is that use of the second network is compensatory. According to this view, the additional network is needed to maintain function as age-related neural changes impair the efficacy of the first, primary network. Those individuals who need to use the second network more are doing so because the first network is more impaired. Thus compensation in this case would be associated with maintenance of function as opposed to improved function.

Testing this idea requires some measure of age-related neural change. The prediction would be that individuals who express the second network are more likely to have age-related neural change that impairs performance of the primary network. In a recent analysis (Table 2, Study 5) (Steffener, Brickman, Rakitin, Gazes, \& Stern, 2008), we used local gray matter brain atrophy as a measure of age-related changes. We used voxel based morphometry to test whether either global atrophy or atrophy specifically in the primary network was related to expression of the secondary network. Global atrophy was not associated with expression of the secondary functional network. However, regional grey matter density in the left pre-central gyrus -- one key area within the primary functional network -- was associated with increased secondary 
network utilization. Further, variability of gray matter density in the left pre-central gyrus was correlated with age only in the elder group. These observations are consistent with the following scenario: as age-related neural changes affect the primary network used by young and old when performing this task, the older increasingly recruit an alternate network. Those that rely more on this alternate network can still perform the task, but do so more poorly. This result is consistent with neural compensation, in that age-related atrophy in the primary network induced the older participants to recruit additional neural resources (the second network) in order to maintain task performance, albeit at a lower level. Followup studies are needed to confirm the compensatory role of the second network. For example, one could explore the effect on elders' performance of TMS applied to the secondary network. Another approach would be to use a combination of TMS and fMRI in a study of young adults while performing this task. One might then be able to determine if TMS applied to a key node to the primary network (e.g. the left precentral gyrus) results in the younger subjects recruiting the secondary network.

Another analytic strategy for demonstrating the presence of neural compensation was taken in a PET study (Stern et al., 2005) which examined the nonverbal serial recognition task in 17 young adults and 19 healthy elderly adults (Table 2, Study 6). The cognitive reserve variable that we used in this study was a factor score that summarized years of education and scores on two IQ indices, the NART and WAIS-R vocabulary score. We used a version of OrT CVA to identify a set of functionally connected regions that: 1) changed in expression from the low demand to the titrated demand conditions, and 2) were differentially expressed by the young and elderly subjects. That is, this analysis specifically sought out a covariance pattern that was differentially expressed by the two groups and therefore represents altered task-related activation in the older subjects. The regions most active in the brain network we identified in this manner consisted of right hippocampus, posterior insula, thalamus and right and left operculum. Concomitant deactivation was recorded in the right lingual gyrus, inferior parietal lobe, association cortex, left posterior cingulate, and right and left calcarine cortex.

Since young subjects operate without the burden of any age-related physiologic changes, we began by evaluating expression of this network in the young group. The mean expression of this network in the young subjects was lower than that in the old subjects, but the correlation between network expression and reserve was positive. Thus, in the transition from low to titrated demand condition, the higher their level of $\mathrm{CR}$, the more young subjects increased their activation in regions with positive loadings, with concomitantly decreased activation in regions with negative loadings. The differential utilization of this topography by young subjects as a function of $\mathrm{CR}$ is consistent with our prediction of the behavior of a neural reserve network.

In the elders, the correlation between the $\mathrm{CR}$ index and their expression of the age-related topography was negative. That is, the higher their level of CR, the more old subjects increased their activation in regions with negative loadings and decreased their activation in regions with positive loadings in the transition from low to titrated demand condition. Thus, as in the young subjects, individual differences in elder subjects' network expression in response to increased task demand correlated with a measure of CR. However, the direction of this relationship was the opposite of that seen in the young subjects.

Since the young subjects have no age related neural changes, we can speculate that the different relationship between CR and topographic expression in the two groups is due to some agerelated physiological change in the older subjects. As a response to these changes, perhaps as a function of longer-term brain adaptation, the older subjects make use of an altered network, causing the activation of the regions captured in the covariance pattern to switch sign. This results in higher CR being associated with increased utilization of some brain areas with more positive network expression in one group, and more negative expression in the other. The age- 
related changes in network expression are thus most consistent with our definition of neural compensation.

\section{CR-specific activation}

To this point, this review has focused on how variability in task-related activation might give clues to the neural implementation of CR. This approach relies on the idea that CR can be mediated by differential expression of the networks typically used to mediate task performance, or by differential recruitment of new compensatory networks in the face of brain damage. Since this approach centers on the networks that directly underlie task performance, it does not address an alternate concept of how $\mathrm{CR}$ is neurally mediated. The epidemiologic data suggest that $\mathrm{CR}$ allows someone to maintain functioning across a wide range of tasks for a longer time in the face of aging or $\mathrm{AD}$ pathology. This suggests that the brain networks subserving cognitive reserve are not equivalent to those required to perform any one particular task. Rather, it is quite likely that a more general "cognitive reserve network" would be elicited by many tasks. By subserving some general as opposed to task-specific function, cognitive reserve might allow someone to cope with pathology and maintain effective functioning for a longer period of time across many domains.

Since the nature and underlying cognitive operations of such a network are not clear, the task at hand might be described as studying the relationship between task-related brain activation and proxies for cognitive reserve, as opposed to the relationship to performance of the task itself. We have addressed this question by using CR proxies as covariates in our imaging analyses. In each case, we tried to identify patterns of load-related activation that are expressed as a function of CR. This allows us to study what aspects of task-related activation vary as a function of CR, whether or not this activation would be identified if we simply looked at taskrelated activation itself. Further, in one study we sought to determine whether such CR-related activation could be noted across two tasks with different processing demands. That is, could we derive a generic CR network that might be operating across multiple tasks?

Two analyses of data from our continuous nonverbal recognition task investigated activation that correlated with measures of CR. The first study (Table 2, Study 7) (Stern et al., 2003) used 19 healthy young adults between the ages of 18 and 30. The raw score of the NART was used as a proxy measure for cognitive reserve. A GLM analysis sought brain areas where the change in fMRI response amplitude from low to titrated demand conditions correlated with an individual subject's NART scores. During the study phase of the task, positive correlations between load-related activation and NART were seen in left middle frontal gyrus and negative correlations were seen at right superior frontal gyrus, middle frontal gyrus, precentral gyrus, medial frontal gyrus, and insula. We also found brain areas that showed correlations between task-related activation and NART scores during the recognition phase of the task. In summary, the primary finding of this study was that, both during study and during subsequent retrieval, brain areas were noted where there was a systematic relationship between CR and brain activation. These correlations point to aspects of processing that differ as a function of CR in healthy young adults. This study can be contrasted with Study 2 described above. Recall that in Study 2 we sought to identify a network that increased in expression from the low to the titrated demand condition. This network was presumed to represent aspects of task processing that change with task load. We treated individual differences in increase in network expression as a measure differential efficiency and related these changes $t$ measures if CR. Thus that study focused on how the neural substrates of performing the task itself and how they may be related to CR. In contrast, the current study directly explored the neural substrates of CR itself by asking whether there are aspects of load related activation that correlate with CR, whether or not they directly mediate task performance. Another study that used a similar approach (Table 2 , Study 8 ) was described above in the section entitled Different networks underlying task 
performance in young and old. That study used the same nonverbal serial learning task, PET as the imaging modality, and both old and young subjects were included. As described above, that study used a GLM approach that searched for voxels in which there was a correlation between the CR measure and the change in activation from the low to the titrated condition. We found brain areas in young and old where such correlations were observed. Again this approach is aimed at identifying activation associated with $\mathrm{CR}$ as opposed to that associated with task performance.

The final study reviewed here (Table 2, Study 9) (Stern et al., 2007) sought to determine whether there is a generic network that subserves CR across multiple tasks. Our strategy was to see if we could find a single network that showed increased load-related activation as a function of $\mathrm{CR}$ across two tasks with differing cognitive processing demands. Young and elder subjects were scanned with fMRI while performing either the letter or the shape Sternberg task. Load-dependent fMRI signal corresponding to each trial component (i.e., stimulus presentation, retention delay, and probe) and task (letter or shape) were regressed onto putative $\mathrm{CR}$ variables. We then used MLM to summarize the imaging data $-\mathrm{CR}$ relationships. We wished to determine if there were patterns of CR-related brain activity whose latent predictors had similar contributions from both the letter and shape tasks. Such a pattern, expressed across two tasks with divergent processing demands, would be a likely candidate for a generic neural substrate underlying CR. We identified a pattern like this in the young group: a spatial pattern expressed during the stimulus presentation phase manifested similar relationships between CR and load-related activation across both the letter and shape WM tasks. Thus, in the young subjects we identified a common CR network that was expressed across both tasks. Elders expressed the network in a manner similar to the younger subjects when performing the letter task, but not the shape task.

The analysis did not concern itself with whether differential expression of this CR-related spatial pattern was associated with better or worse performance. In fact, we explicitly eliminated the possibility of such a relationship in the analytic design. This reduces the chance that CR-related network expression is influenced by differences in performance across individuals. We relied on the effortful processing in these tasks (by looking at load-related activation) to elicit CR-related networks, independent of performance. This means that a highCR young person, although badly performing in a high-demand task, will still show a pure instantiation of the CR-network. The inference that we wish to draw is that this network might represent the neural instantiation of $\mathrm{CR}$, or alternately that the ability to invoke this network might underlie the benefits that CR imparts. Because this pattern reflects a CR-related network that is used by healthy individuals it meets the proposed criteria for neural reserve.

It is of interest that in the elders, pattern expression was not consistent across the two tasks. Notably, the directionality of pattern expression was similar to that in the young subjects for the letter but not the shape task. Follow up studies are needed to test the idea that the CR-related pattern can be used by elders in the simpler letter task but not in the more challenging shape task.

The common CR pattern noted here consisted of bilateral superior frontal gyrus (BA 10), left medial frontal gyrus 9, right medial frontal gyrus $(6,8)$, and left Middle frontal gyrus (8). Many of the areas included in the common CR pattern here have been noted in studies of control processes such as task switching (Braver, Reynolds, \& Donaldson, 2003; Wager, Jonides, \& Reading, 2003), as well as in some studies of working memory (Wager \& Smith, 2006). In the fMRI study described above (Table 2, Study 7), which used the nonverbal serial recognition task (Stern et al., 2003), we found several of the same areas noted here were differentially activated as a function of CR during both the encoding and retrieval phases of the task. As in the current study, increased expression of these areas was associated with higher measured 
CR. These consistent findings across studies and tasks provide a preliminary suggestion that control processes may be an important component of some aspects of CR.

This study raises two important sets of questions that must be addressed in the future. First, it will be of interest to see if expression of this CR-related network by younger subjects can be detected during the performance of tasks not used in the current study. If the network is expressed across multiple tasks it would support the idea that it mediates a general feature of CR. In the future, it will be even more important to determine whether differential expression of any putative CR pattern actually imparts reserve against the neural effects of aging. One way to address this question would be to measure expression of such a network in a set of younger subjects and then follow them over time, with the prediction that higher expression will predict slower progression of age-related cognitive changes.

\section{Conclusions}

There is strong epidemiologic evidence for the idea that cognitive reserve mediates between brain changes or pathology and the clinical manifestation of that pathology. The concept of cognitive reserve strongly relies on the idea that there can be individual differences in how tasks are processed that can allow some people to cope better than others with brain changes in general and aging in particular. I have tried to map out a systematic approach for exploring the neural implementation of CR. I do not view the studies presented here as definitively identifying the neural substrates of CR. Rather, they help to establish methodologies for exploring these neural substrates. These approaches acknowledge that reserve may be mediated in many different ways, and the results presented here can be seen as supporting the suppositions presented above about how CR might be neurally mediated. Neural reserve recognizes that old and young individuals may use the same networks to mediate task performance, albeit with different levels of efficiency and capacity. This inter-individual variability is present even within these two groups, and differences in neural reserve may be one mechanism underlying CR. On the other hand, there are clearly situations where older individuals adopt networks that are not used by younger subjects, presumably in response to age-related neural changes. These alternate networks may represent neural compensation. The degree of compensation can again vary across individuals in its expression and success. Evaluating neural compensation in imaging studies is often hampered by the difficulty of measuring the underlying brain changes that induce this compensation. Simply relating degree of expression of compensatory activation to performance is not sufficient. As demonstrated above, compensatory activation can be accompanied by both better and poorer performance. In either case CR might also be mediated in part via neural compensation.

Finally, CR may not be solely mediated via variability in the expression of the specific brain networks associated with the task at hand; there may be one or more general, reserve-related networks whose expressions are associated with $\mathrm{CR}$ across many different tasks. This translates into the question of whether CR is task-specific or represents some generalized cognitive function that is associated with the performance of multiple tasks. The epidemiologic evidence suggests that a set of lifetime exposures translates into a cognitive reserve that mediates the brain changes associated with aging or $\mathrm{AD}$. This makes it attractive to speculate that a general reserve-related network may be identified.

Determining whether or not there is a generalized CR network is important for considering whether it will be possible to intervene to impart increased CR and thereby slow the effects of advancing age or $\mathrm{AD}$ pathology. Research to date suggests that cognitive training benefits only the task used in training itself and does not generalize to other tasks or behaviors. Better understanding of the neural mediation of CR may provide suggestions for targets and approaches for increasing CR and improving performance across a wider range of tasks. 
The possibility of using an imaging approach to measure $\mathrm{CR}$ could also have other practical implications. Imaging could be used as a meaningful outcome in cognitive interventions. Imaging CR would also be very useful for understanding any aged individual's true clinical status, which would be a combination of underlying age-related (or Alzheimer's diseaserelated) brain changes and that individuals' CR in the face of those changes. Two individuals who appear the same clinically could differ widely on these underlying measures. This approach to characterizing clinical severity would have strong implications for prognosis and treatment.

The concept of CR first emerged from epidemiologic observations. The desire to understand the neural basis of $\mathrm{CR}$ has been a motivating factor for functional imaging studies that may contribute to our understanding of the brain-behavior changes that occur with aging. Finally, studies of CR may point the way to successful interventions that can help maintain successful aging and slow the onset of dementia. Similar applications of the concept of CR to other types of brain pathology have begun to yield similar fruit. While CR is an intuitively attractive concept, hopefully this review makes it clear that its application to applied research requires theoretical and methodologic consideration. With the proper research designs, the concept of CR provides a frame for generating powerful and empirically testable research ideas.

\section{Acknowledgments}

This work was supported by a grant from the National Institutes on Aging (RO1 AG26158).

\section{Reference List}

Albert MS, Jones K, Savage CR, Berkman L, Seeman T, Blazer D, et al. Predictors of cognitive change in older persons: MacArthur studies of successful aging. Psychology and Aging 1995;10:578-589. [PubMed: 8749585]

Albert SM, Teresi JA. Reading ability, education, and cognitive status assessment among older adults in Harlem, New York City. American Journal of Public Health 1999;89:95-97. [PubMed: 9987476]

Alexander GE, Furey ML, Grady CL, Pietrini P, Mentis MJ, Schapiro MB. Association of premorbid function with cerebral metabolism in Alzheimer's disease: Implications for the reserve hypothesis. American Journal of Psychiatry 1997;154:165-172. [PubMed: 9016263]

Barnett JH, Salmond CH, Jones PB, Sahakian BJ. Cognitive reserve in neuropsychiatry. Psychological Medicine 2006;36:1053-1064. [PubMed: 16854246]

Bennett DA, Wilson RS, Schneider JA, Evans DA, Mendes De Leon CF, Arnold SE, et al. Education modifies the relation of $\mathrm{AD}$ pathology to level of cognitive function in older persons. Neurology 2003;60(12):1909-1915. [PubMed: 12821732]

Braver TS, Reynolds JR, Donaldson DI. Neural mechanisms of transients and sustained cognitive control during task switching. Neuron 2003;39:713-726. [PubMed: 12925284]

Brown J, Cooper-Kuhn CM, Kemperman G, van Praag H, Winkler J, Gage FH. Enriched environment and physical activity stimulate hippocampal but not olfactory bulb neurogenesis. EUROPEAN JOURNAL OF NEUROSCIENCE 2003;17:2042-2046. [PubMed: 12786970]

Butler SM, Ashford JW, Snowdon DA. Age, education, and changes in the Mini-Mental State Exam scores of older women: findings from the Nun Study. Journal of the American Geriatrics Society 1996;44:675-681. [PubMed: 8642159]

Cabeza R. Hemispheric asymmetry reduction in older adults: The HAROLD model. Psychology \& Aging 2002;17:85-100. [PubMed: 11931290]

Chodosh J, Reuben DB, Albert MS, Seeman TE. Predicting cognitive impairment in high-functioning community-dwelling older persons: MacArthur Studies of Successful Aging. Journal of the American Geriatrics Society 2002;50:1051-1060. [PubMed: 12110065]

Christensen H, Korten AE, Jorm AF, Henderson AS, Jacomb PA, Rodgers B, et al. Education And Decline In Cognitive Performance: Compensatory But Not Protective. International Journal of Geriatric Psychiatry 1997;12:323-330. [PubMed: 9152716] 
Colsher PL, Wallace RB. Longitudinal application of cognitive function measures in a defined population of community-dwelling elders. Annals of Epidemiology 1991;1:215-30. [PubMed: 1669503]

Craik FI. Brain-behavior relations across the lifespan: a commentary. Neurosci Biobehav Rev 2006;30:885-892. [PubMed: 16919731]

DeCarli C, Atack JR, Ball MJ, Kay JA, Grady CL, Fewster P, et al. Post-mortem regional neurofibrillary tangle densities but not senile plaque densities are related to regional cerebral metabolic rates for glucose during life in Alzheimer's disease patients. Neurodegeneration 1992;1:113-121.

Evans DA, Beckett LA, Albert MS, Hebert LE, Scherr PA, Funkenstein HH, et al. Level of education and change in cognitive function in a community population of older persons. Annals of Epidemiology 1993;3:71-77. [PubMed: 8287159]

Farinpour R, Miller EN, Satz P, Selnes OA, Cohen BA, Becker JT, et al. Psychosocial risk factors of HIV morbidity and mortality: findings from the Multicenter AIDS Cohort Study (MACS). Journal of Clinical and Experimental Neuropsychology 2003;25:654-670. [PubMed: 12815503]

Farmer ME, Kittner SJ, Rae DS, Bartko JJ, Regier DA. Education and change in cognitive function: The epidemiologic catchment area study. Annals of Epidemiology 1995;5:1-7. [PubMed: 7728280]

Friedland RP, Brun A, Bundinger TF. Pathological and positron emission tomographic correlations in Alzheimer's disease. Lancet 1985:1-228.

Grady CL, Maisog JM, Horwitz B, et al. Age-related changes in cortical blood flow activation during visual processing of faces and location. J Neurosci 1994;14:1450-1462. [PubMed: 8126548]

Grober E, Sliwinski M. Development and validation of a model for estimating premorbid verbal intelligence in the elderly. Journal of Clinical and Experimental Neuropsychology 1991;13:933-949. [PubMed: 1779032]

Habeck C, Hilton HJ, Zarahn E, Flynn J, Moeller JR, Stern Y. Relation of cognitive reserve and task performance to expression of regional covariance networks in an event-related fMRI study of nonverbal memory. Neuroimage 2003;20:1723-1733. [PubMed: 14642482]

Habeck C, Krakauer JW, Ghez C, Sackeim HA, Eidelberg D, Stern Y, et al. A new approach to spatial covariance modeling of functional brain imaging data: Ordinal trend analysis. Neural Computation. in press

Habeck C, Rakitin BC, Moeller J, Scarmeas N, Zarahn E, Brown T, et al. An event-related fMRI study of the neural networks underlying the encoding, maintenance, and retrieval phase in a delayed-matchto-sample task. Brain Res Cogn Brain Res 2005b;23:207-20. [PubMed: 15820629]

Hall CB, Derby C, LeValley A, Katz MJ, Verghese J, Lipton RB. Education delays accelerated decline on a memory test in persons who develop dementia. Neurology 2007;69:1657-1664. [PubMed: 17954781]

Helzner EP, Scarmeas N, Cosentino S, Portet F, Stern Y. Leisure activity and cognitive decline in incident Alzheimer disease. Arch Neurol 2007;64:1749-1754. [PubMed: 18071038]

Holtzer R, Rakitin BC, Steffener J, Flynn J, Kumar A, Stern Y. Age effects on load-dependent brain activations in working memory for novel material. Brain Research. in press

Ince PG. Pathological correlates of late-onset dementia in a multicenter community-based population in England and Wales. Lancet 2001;357:169-175. [PubMed: 11213093]

Katzman R. Education and the prevalence of dementia and Alzheimer's disease. Neurology 1993;43:1320. [PubMed: 8423876]

Kesler SR, Adams HF, Blasey CM, Bigler ED. Premorbid intellectual functioning, education, and brain size in traumatic brain injury: An investigation of the cognitive reserve hypothesis. Applied Neuropsychology 2003;10:153-162. [PubMed: 12890641]

Lazarov O, Robinson J, Tang YP, Hairston IS, Korade-Mirnics Z, Lee VM, et al. Environmental enrichment reduces Abeta levels and amyloid deposition in transgenic mice. Cell 2005;120:701-13. [PubMed: 15766532]

Li SC, Lindenberger U, Sikstrom S. Aging cognition: from neuromodulation to representation. Trends Cogn Sci 2001;5:479-486. [PubMed: 11684480]

Lyketsos CG, Chen LS, Anthony JC. Cognitive Decline in Adulthood: An 11.5-Year Follow-Up of the Baltimore Epidemiologic Catchment Area Study. American Jounal of Psychiatry 1999;156:58-65. 
Madden DJ, Turkington TG, Provenzale JM, Denny LL, Hawk TC, Gottlob LR, et al. Adult age differences in the functional neuroanatomy of verbal recognition memory. Hum Brain Mapp 1999;7:115-35. [PubMed: 9950069]

Manly JJ, Schupf N, Tang MX, Stern Y. Cognitive decline and literacy among ethnically diverse elders. Journal of Geriatic Psychiatry \& Neurology 2005;18:213-217.

Manly JJ, Touradji P, Tang MX, Stern Y. Literacy and Memory Decline Among Ethnically Diverse Elders. Journal of Clinical and Experimental Neuropsychology 2003;5:680-690. [PubMed: 12815505]

McGeer EG, McGeer PL, Harrop R, Akiyama H, Kamo H. Correlations of regional postmortem enzyme activities with premortem local glucose metabolic rates in Alzheimer's disease. J Neurosci Res 1990;27:612-619. [PubMed: 2079721]

Mortel KF, Meyer JS, Herod B, Thornby J. Education and occupation as risk factors for dementia of the Alzheimer and ischemic vascular types. Dementia 1995;6:55-62. [PubMed: 7728220]

Nelson HE, O'Connell A. Dementia: The estimation of premorbid intelligence levels using the National Adult Reading Test. Cortex 1978;14:234-244. [PubMed: 679704]

Perneczky R, Drzezga A, ehl-Schmid J, Schmid G, Wohlschlager A, Kars S, et al. Schooling mediates brain reserve in Alzheimer's disease: findings of fluoro-deoxyglucose-positron emission tomography. J Neurol Neurosurg Psychiatry 2006;77:1060-1063. [PubMed: 16709580]

Rajah MN, D'Esposito M. Region-specific changes in prefrontal function with age: a review of PET and fMRI studies on working and episodic memory. Brain 2005;128:1964-1983. [PubMed: 16049041]

Reuter-Lorenz P. New visions of the aging mind and brain. Trends Cogn Sci 2002;6:394. [PubMed: 12200182]

Richards M, Sacker A. Lifetime antecedents of cognitive reserve. Journal of Clinical and Experimental Neuropsychology 2003;25:614-624. [PubMed: 12815499]

Rocca WA, Bonaiuto S, Lippi A, Luciani P, Turtu F, Cavarzeran F, et al. Prevalence of clinically diagnosed Alzheimer's disease and other dementing disorders: A door-to-door survey in Appignano, Macerata Province, Italy. Neurology 1990;40:626-631. [PubMed: 2320236]

Rypma B, Prabhakaran V, Desmond JE, Glover GH, Gabrieli JD. Load-dependent roles of frontal brain regions in the maintenance of working memory. Neuroimage 1999;9:216-226. [PubMed: 9927550]

Satz P. Brain reserve capacity on symptom onset after brain injury: A formulation and review of evidence for threshold theory. Neuropsychology 1993;7:273-295.

Scarmeas N, Albert SM, Manly JJ, Stern Y. Education and rates of cognitive decline in incident Alzheimer's disease. Annals of Neurology. in press

Scarmeas N, Levy G, Tang MX, Manly J, Stern Y. Influence of leisure activity on the incidence of Alzheimer's disease. Neurology 2001;57:2236-2242. [PubMed: 11756603]

Scarmeas N, Zarahn E, Anderson KE, Habeck CG, Hilton J, Flynn J, et al. Association of life activities with cerebral blood flow in Alzheimer disease - Implications for the cognitive reserve hypothesis. Archives of Neurology 2003a;60:359-365. [PubMed: 12633147]

Scarmeas N, Zarahn E, Anderson KE, Hilton HJ, Flynn J, Van Heertum RL, et al. Cognitive reserve modulates functional brain responses during memory tasks: a PET study in healthy young and elderly subjects. Neuroimage 2003b;19:1215-1227. [PubMed: 12880846]

Snowdon DA, Ostwald SK, Kane RL. Education, survival and independence in elderly Catholic sisters, 1936-1988. American Journal of Epidemiology 1989;130:999-1012. [PubMed: 2816907]

Steffener J, Brickman AM, Rakitin BC, Gazes Y, Stern Y. The impact of structure on age-related changes in working memory functional activity. Brain Imaging and Behavior. in press

Stern Y. What is cognitive reserve? Theory and research application of the reserve concept. Journal of the International Neuropsychological Society 2002;8:448-460. [PubMed: 11939702]

Stern, Y. Imaging cognitive reserve. In: Stern, Y., editor. Cognitive Reserve: Theory and Applications. New York: Taylor \& Francis; 2007. p. 251-264.

Stern Y, Albert S, Tang MX, Tsai WY. Rate of memory decline in AD is related to education and occupation: Cognitive reserve? Neurology 1999;53:1942-1957. [PubMed: 10599762] 
Stern Y, Alexander GE, Prohovnik I, Mayeux R. Inverse relationship between education and parietotemporal perfusion deficit in Alzheimer's disease. Annals of Neurology 1992;32:371-375. [PubMed: 1416806]

Stern Y, Alexander GE, Prohovnik I, Stricks L, Link B, Lennon MC, et al. Relationship between lifetime occupation and parietal flow: Implications for a reserve against Alzheimer's disease pathology. Neurology 1995;45:55-60. [PubMed: 7824135]

Stern Y, Gurland B, Tatemichi TK, Tang MX, Wilder D, Mayeux R. Influence of education and occupation on the incidence of Alzheimer's disease. Journal of the American Medical Association 1994;271:1004-1010. [PubMed: 8139057]

Stern Y, Habeck C, Moeller J, Scarmeas N, Anderson KE, Hilton HJ, et al. Brain networks associated with cognitive reserve in healthy young and old adults. Cereb Cortex 2005;15:394-402. [PubMed: 15749983]

Stern Y, Tang MX, Denaro J, Mayeux R. Increased risk of mortality in Alzheimer's disease patients with more advanced educational and occupational attainment. Annals of Neurology 1995;37:590-595. [PubMed: 7755353]

Stern Y, Zarahn E, Habeck C, Holtzer R, Rakitin BC, Kumar A, et al. A Common Neural Network for Cognitive Reserve in Verbal and Object Working Memory in Young but not Old. Cereb Cortex. 2007

Stern Y, Zarahn E, Hilton HJ, Delapaz R, Flynn J, Rakitin B. Exploring the neural basis of cognitive reserve. Journal of Clinical and Experimental Neuropsychology 2003;5:691-701. [PubMed: 12815506]

Valenzuela MJ, Sachdev P. Brain reserve and dementia: a systematic review. Psychological Medicine 2005;35:1-14.

van Praag H, Shubert T, Zhao C, Gage FH. Exercise enhances learning and hippocampal neurogenesis in aged mice. Journal of Neuroscience 2005;25:8680-8685. [PubMed: 16177036]

Wager TD, Jonides J, Reading S. Neuroimaging of shifting attention: a meta-analysis. Neuroimage 2003;22:1679-1693. [PubMed: 15275924]

Wager TD, Smith EE. Neuroimaging studies of working memory: A meta-analysis. Cognitive, Affective, \& Behavioral Neuroscience 2006;3:255-274.

Willerman L, Schultz R, Rutledge JN, Bigler ED. In vivo brain size and intelligence. Intelligence 1991;15:223-228.

Worsley KJ, Poline JB, Friston KJ, Evans AC. Characterizing the response of PET and fMRI data using multivariate linear models. Neuroimage 1997;6:305-19. [PubMed: 9417973]

Zarahn E, Rakitin B, Abela D, Flynn J, Stern Y. Age-related changes in brain activation during a delayed item recognition task. Neurobiol Aging 2007;28:784-798. [PubMed: 16621168] 


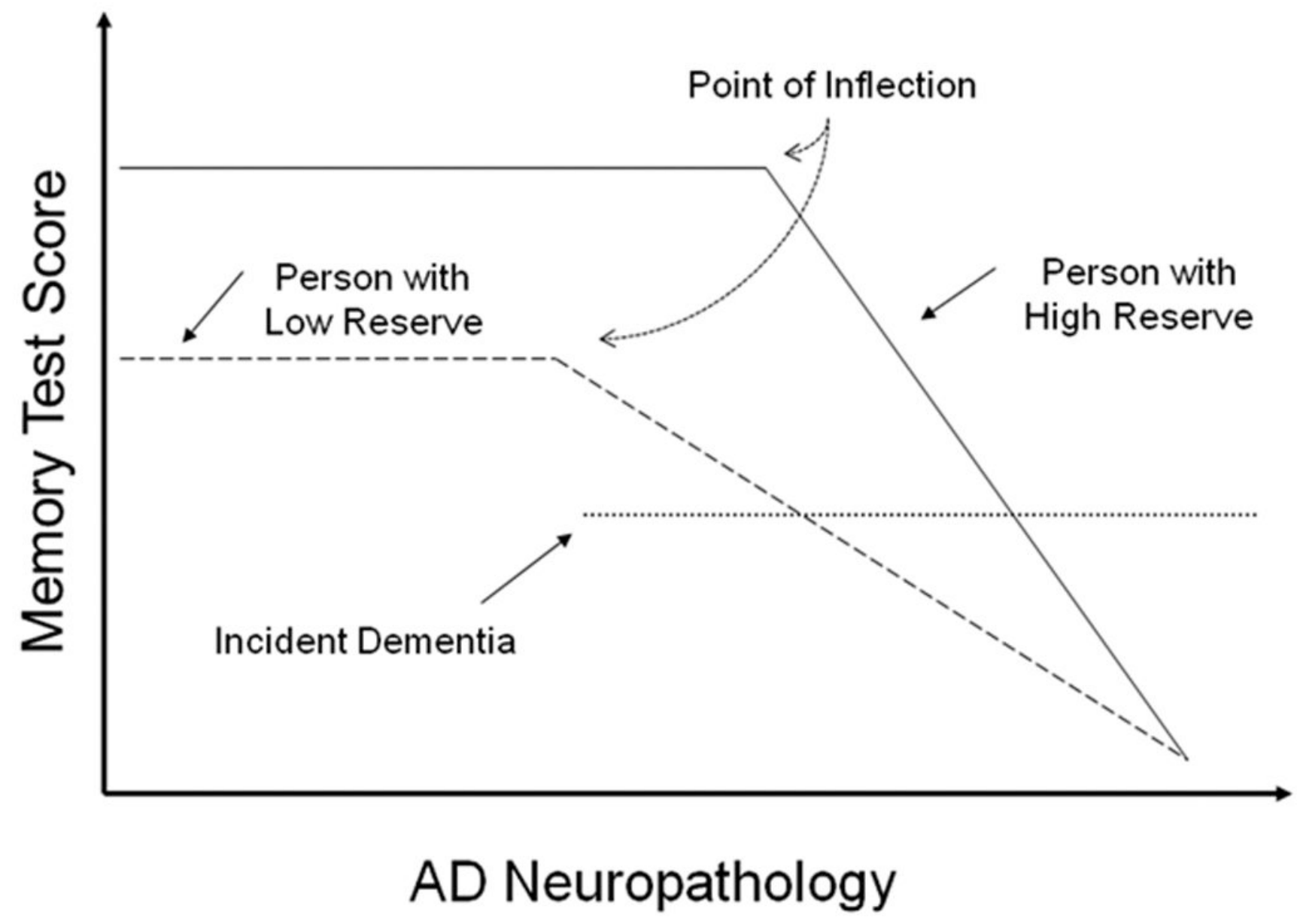

Figure 1.

Theoretical illustration of how cognitive reserve may mediate between AD pathology and its clinical expression. We assume that AD pathology slowly increases over time, and this is graphed on the $\mathrm{x}$-axis. The $\mathrm{y}$-axis represents cognitive function, in this case memory performance. Assuming that AD pathology increases over time at the same rate in two individuals with high and low reserve, the following predictions can be made about the individual with high CR: 1) the point of inflection, where memory begins to be affected AD pathology will be later; 2) clinical diagnostic criteria for AD be reached later, when pathology is more severe; 3 ) at any level of memory performance, AD pathology will be more severe; 4) after the point of inflection, clinical progression will be more rapid. 


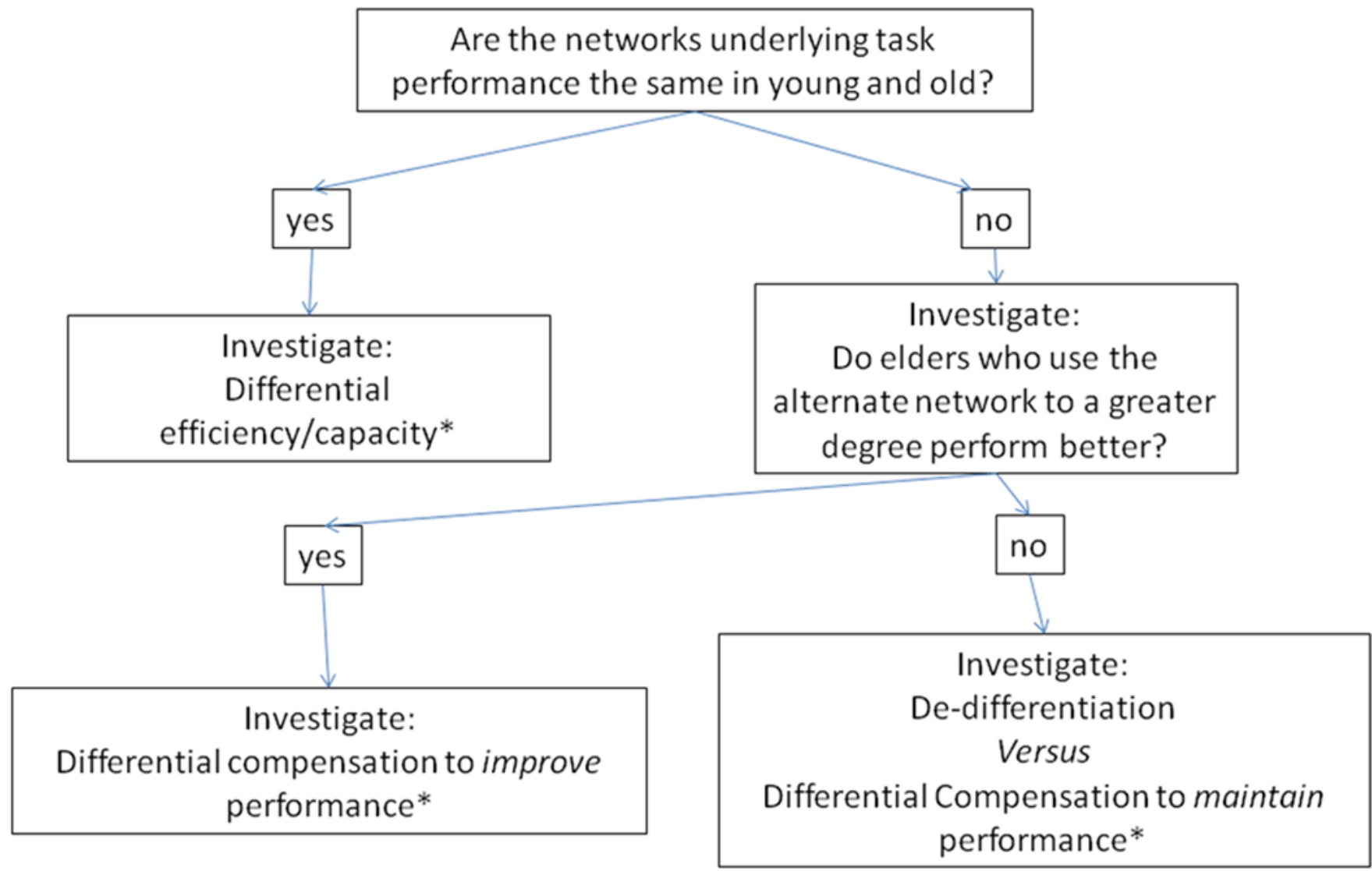

*In each instance, investigate: How do individual differences relate to proxy measures of cognitive reserve

Figure 2.

A scheme for exploring and comparing task related activation and young and old with the goal of understanding cognitive reserve. The same approach would apply to any condition that could potentially disrupt brain function. Each step in this scheme is discussed in the text. 


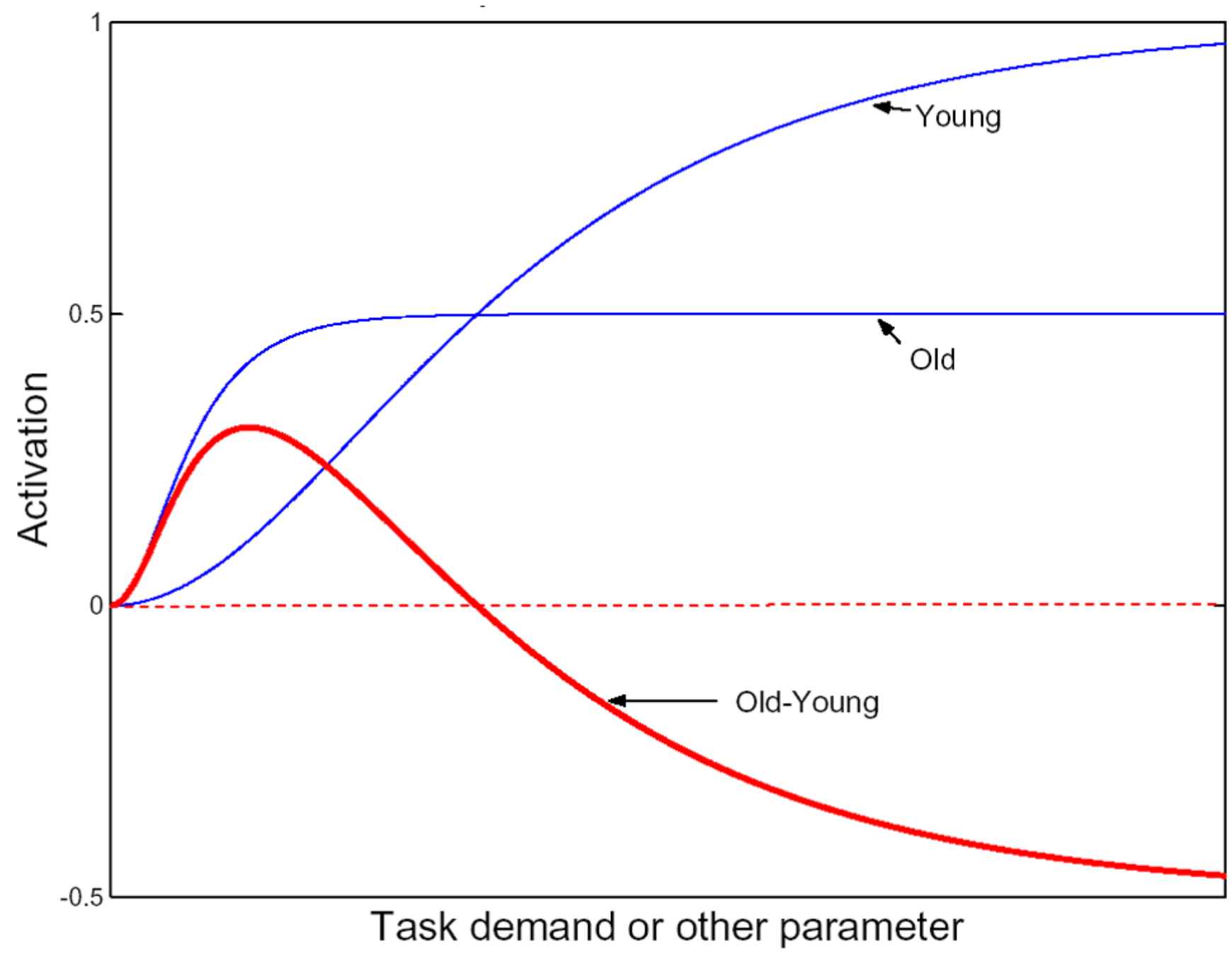

Figure 3.

Hypothetical relationship between task demand and activation in old and young. On the $\mathrm{X}$ axis is increasing task demand, referring to a within-subject manipulation where the difficulty of the task is increased in a parametric manner. The $\mathrm{Y}$ axis represents task-related activation either at one particular brain location or throughout some brain network. The figure demonstrates hypothesized curves relating task demand to task-related activation in young and old individuals. The rate of rise in the curve is an index of the efficiency of the system. The asymptote of the curves could indicate the capacity of the system. Note in the illustration that at relatively low task demand, greater activation might be seen in old people than in young people. Conversely, at greater task demand, greater activation might be seen in the young subjects than in the old. 


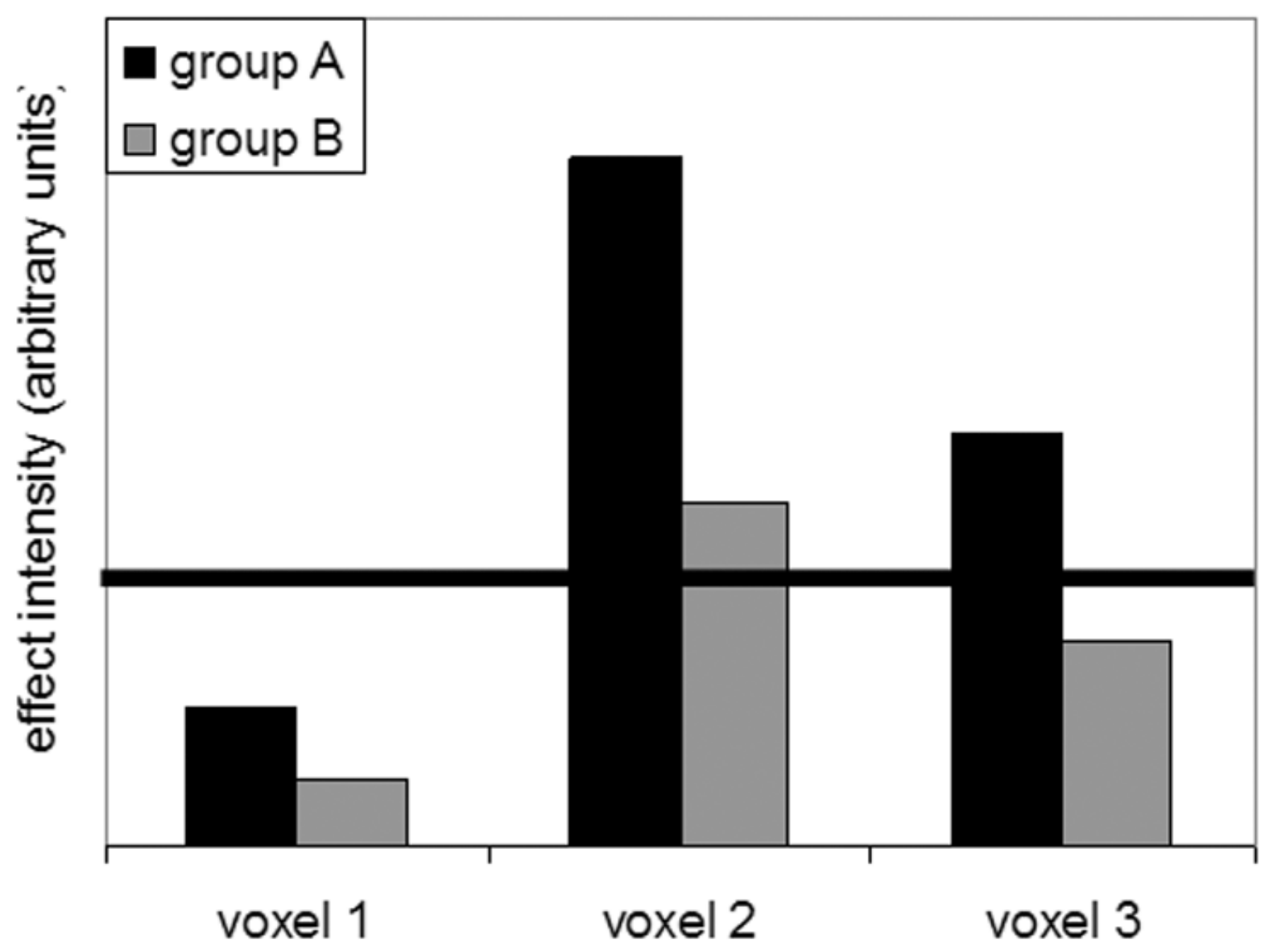

Figure 4.

Example of two groups expressing the same pattern of activation across three voxels (or brain areas) but to different degrees. When comparing thresholded maps between groups, as in standard voxel-wise GLM analyses, one would not appreciate the fact that both groups express the same pattern of activation. Further, one might incorrectly conclude activation of voxel 3 is unique to group A. Specific covariance analyses such as MLM and OrT CVA are designed to identify patterns such as these and compare their expression across groups. 
Table 1

Working definitions of key concepts

Brain Reserve: Individual differences in the brain itself allow some people to cope better than others with brain pathology. These differences can be quantitative, such as larger brain, more neurons, or synapses. In addition, life experience can influence brain anatomy via neurogenesis, angiogenesis, promoting resistance to apoptosis, and up-regulating compounds that promote neural plasticity.

Cognitive Reserve: Individual differences in how people process tasks allow some to cope better than others with brain pathology.

Neural Reserve: Inter-individual variability - perhaps in the form of differing efficiency, capacity, or flexibility - in the brain networks or cognitive paradigms that underlie task performance in the healthy brain. An individual whose networks are more efficient, have greater capacity, or are more flexible might be more capable of coping with the disruption imposed by brain pathology.

Neural Compensation: Inter-individual variability in the ability to compensate for brain pathology's disruption of standard processing networks by using brain structures or networks not normally used by individuals with intact brains. This compensation may help maintain or improve performance. 


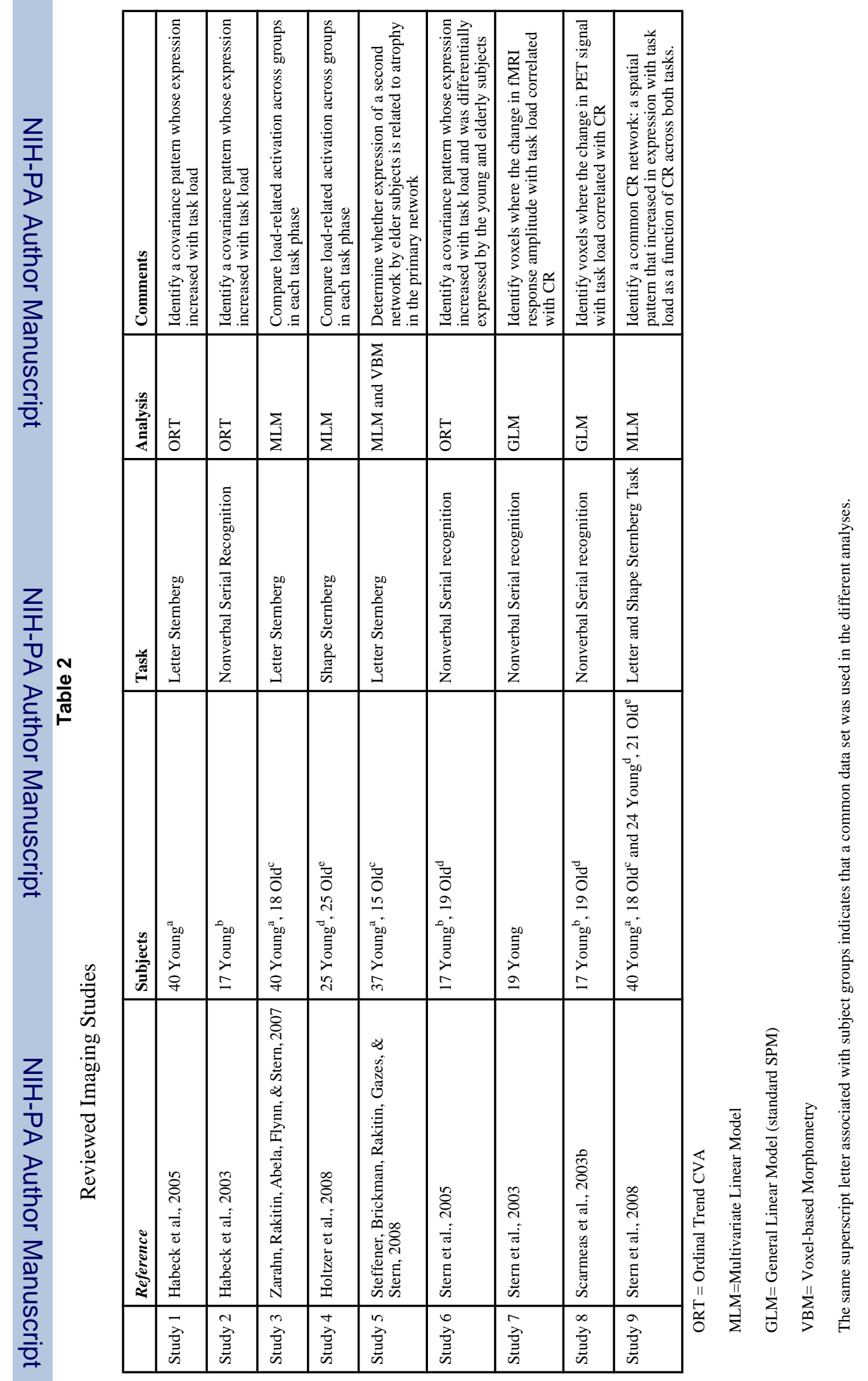

